\title{
SEVEN-DIMENSIONAL CONSIDERATIONS OF EINSTEIN'S CONNECTION. III. THE SEVEN-DIMENSIONAL EINSTEIN'S CONNECTION
}

\author{
MI AE KIM, KEUM SOOK SO, CHUNG HYUN CHO, \\ and KYUNG TAE CHUNG
}

Received 12 April 2001 and in revised form 27 September 2001

\begin{abstract}
The purpose of the present paper is to prove a necessary and sufficient condition for a unique Einstein's connection to exist in 7-g-UFT and to display a surveyable tensorial representation of seven-dimensional Einstein's connection in terms of the unified field tensor, employing the powerful recurrence relations of the third kind obtained in earlier papers. All considerations in this paper are restricted to the first and second classes of $X_{7}$, since the case of the third class, the simplest case, was already studied by many authors.
\end{abstract}

2000 Mathematics Subject Classification: 83E50, 83C05, 58A05.

Einstein's connection in a generalized Riemannian manifold $X_{n}$ has been investigated by many authors for lower-dimensional cases $n=2, \ldots, 6$. In a series of papers, we obtain a surveyable tensorial representation of seven-dimensional Einstein's connection in terms of unified field tensor, with main emphasis on the derivation of powerful and useful recurrence relations which hold in $X_{7}$.

In [2], which we denote by I in the present paper, we gave a brief survey of Einstein's unified field theory and derived the recurrence relations of the first kind which hold in a general $X_{n}$. In [1], which we denote by II in the present paper, we derived a powerful recurrence relations of the second and third kinds which hold in seven-dimensional Einstein's generalized Riemannian manifold $X_{7}$. These relations will be used in the present paper to find a tensorial representation of seven-dimensional Einstein's connection. All considerations in this paper are based on the results and symbolism of I and II. Whenever necessary, they will be quoted in the present paper.

In the following theorem, we prove a necessary and sufficient condition for a unique Einstein's connection to exist in 7-g-UFT.

THEOREM 1 (for the first and second class). A necessary and sufficient condition for the existence and uniqueness of the solution of [1, (2.5) or (2.26)] in 7-g-UFT is given by:

For the first class,

$$
g A B C\left(D^{2}-4 D E+F\right) \neq 0,
$$


where

$$
\begin{aligned}
A= & 1-3 K_{2}+9 K_{4}-27 K_{6}, \\
B= & 1-K_{4}+K_{2} K_{6}-\left(K_{6}\right)^{2}, \\
C= & 1-2 K_{4}-8 K_{6}+\left(K_{4}\right)^{2}-4 K_{2} K_{6}, \\
D= & \left(1-K_{2}+K_{4}-K_{6}\right)^{2} \\
& -4\left[K_{4}-6 K_{6}-\left(K_{4}\right)^{2}+16\left(K_{6}\right)^{2}+10 K_{4} K_{6}+2 K_{2} K_{6}-2 K_{6}\left(K_{2}\right)^{2}\right], \\
E= & \left(2-K_{2}+3 K_{6}\right)\left(3 K_{6}-K_{2} K_{4}\right), \\
F= & 16\left[\left(2-K_{2}+4 K_{6}\right)^{2}+\left(K_{6}\right)^{2}\right]\left[9\left(K_{6}\right)^{2}-6 K_{2} K_{4} K_{6}+\left(K_{4}\right)^{3}+\left(K_{2}\right)^{3} K_{6}\right] \\
& -16 K_{6}\left(2-K_{2}+4 K_{6}\right)\left[-2\left(K_{2}\right)^{3} K_{6}+\left(K_{2}\right)^{2}\left(K_{4}\right)^{2}+6 K_{2} K_{4} K_{6}-2\left(K_{4}\right)^{3}-9\left(K_{6}\right)^{2}\right] .
\end{aligned}
$$

For the second class with the second category,

$$
\begin{aligned}
\left(1+K_{2}\right. & \left.+K_{4}\right)\left(1-K_{4}\right)^{2}\left(1-3 K_{2}+9 K_{4}\right) \\
& \times\left[\left(1-K_{2}-3 K_{4}\right)^{2}-4 K_{4}\left(\left(K_{2}\right)^{2}-4 K_{4}\right)\right] \neq 0 .
\end{aligned}
$$

For the second class with the first category,

$$
\left(1-\left(K_{2}\right)\right)^{2}\left(1-K_{2}\right)\left(1-3 K_{2}\right) \neq 0 .
$$

Proof. For the first class, the symmetric scalars $\underset{x y z}{M}$ defined by [1, (2.27)] take values as in Table 1 , in virtue of [2, (3a)].

It may be easily verified that the product of 3 factors in the first row of Table 1 is $g$ given by [2, (1)], that of the 4 factors in the second row is $1-3 K_{2}+$ $9 K_{4}-27 K_{6}$, that of the 7 factors in the third row is $1-K_{4}+K_{6} K_{2}-\left(K_{6}\right)^{2}$, and that of the 8 factors in the fourth row is $1-2 K_{4}-8 K_{6}+\left(K_{4}\right)^{2}-4 K_{2} K_{6}$. After a lengthy calculation, we obtain the product of the 12 factors in the fifth row as

$$
D^{2}-4 D E+F,
$$

where $D, E$, and $F$ are given by (2). Therefore, our assertion follows in virtue of $[1,(2.28)]$.

The proof of the second class may be obtained easily from (1) and (4) by simply substituting the corresponding conditions of each case.

In the following three theorems, we establish a linear system containing the torsion tensor $S=S_{\omega \lambda \mu}$ of the Einstein's connection, employing the powerful recurrence relations of the third kind obtained in [2, Theorem 5]. 
TABLE 1

\begin{tabular}{|c|c|}
\hline Values of $x, y, z$ & $\underset{x y z}{M}$ \\
\hline Two of $x, y, z$ are 1,2 or 3,4, or 5,6 & $1-\underset{1}{M^{2}}, 1-\underset{3}{M^{2}}, 1-\underset{5}{M^{2}}$ \\
\hline All indices are equal & $1+3 \underset{1}{M^{2}}, 1+\underset{3}{3 M^{2}}, 1+3 M_{5}^{2}, 1$ \\
\hline $\begin{array}{l}\text { At least one of } x, y, z \text { is } 7 \text { and no two } \\
\text { take } 1,2 \text { nor } 3,4 \text { nor } 5,6\end{array}$ & $1 \pm \underset{13}{M M}, 1 \pm \underset{35}{M M}, 1 \pm \underset{51}{M M}, 1$ \\
\hline $\begin{array}{l}\text { No two are equal and no two take } 1,2 \\
\text { nor } 3,4 \text { nor } 5,6\end{array}$ & $\begin{array}{r}1+M M_{3} \pm M M_{5} \pm \underset{5}{M} M_{1} \\
13 M \\
1-M M_{3} \pm M M_{5} \mp M \\
1 M_{5} M \\
\end{array}$ \\
\hline $\begin{array}{l}\text { Two are equal and one of three is } \\
\text { most } 7\end{array}$ & 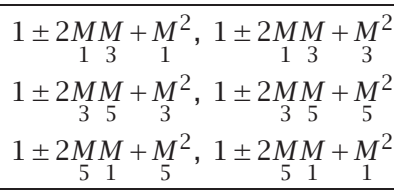 \\
\hline
\end{tabular}

THEOREM 2 (for the first class of 7-g-UFT). The system of [1, (2.26) or (2.5)] is reduced to the following linear system of 66 equations:

$$
\begin{aligned}
& B=S+\stackrel{110}{S}+2 \stackrel{(10) 1}{S}, \\
& \stackrel{(10) 1}{B}=\stackrel{(10) 1}{S}+\stackrel{(21) 1}{S}+\stackrel{(20) 2}{S}+\stackrel{112}{S}, \\
& \stackrel{(12) 1}{B}=\stackrel{(12) 1}{S}+\stackrel{(23) 1}{S}+\stackrel{222}{S}+\stackrel{(13) 2}{S}, \\
& \stackrel{(20) 2}{B}=\stackrel{(20) 2}{S}+\stackrel{(31) 2}{S}+\stackrel{(30) 3}{S}+\stackrel{(21) 3}{S}, \\
& \stackrel{(23) 1}{B}=\stackrel{(23) 1}{S}+\stackrel{(34) 1}{S}+\stackrel{332}{S}+\stackrel{(24) 2}{S}, \\
& \stackrel{(13) 2}{B}=\stackrel{(13) 2}{S}+\stackrel{(24) 2}{S}+\stackrel{(23) 3}{S}+\stackrel{(14) 3}{S}, \\
& \stackrel{(30) 3}{B}=\stackrel{(30) 3}{S}+\stackrel{(41) 3}{S}+\stackrel{(40) 4}{S}+\stackrel{(31) 4}{S}, \\
& \stackrel{(12) 3}{B}=\stackrel{(12) 3}{S}+\stackrel{(23) 3}{S}+\stackrel{224}{S}+\stackrel{(13) 4}{S}, \\
& \stackrel{(34) 1}{B}=\stackrel{(34) 1}{S}+\stackrel{(45) 1}{S}+\stackrel{442}{S}+\stackrel{(35) 2}{S}, \\
& \stackrel{(24) 2}{B}=\stackrel{(24) 2}{S}+\stackrel{(35) 2}{S}+\stackrel{(34) 3}{S}+\stackrel{(25) 3}{S}, \\
& \stackrel{(24) 3}{B}=\stackrel{(24) 3}{S}+\stackrel{(43) 3}{S}+\stackrel{334}{S}+\stackrel{(24) 4}{S},
\end{aligned}
$$




$$
\begin{aligned}
& \stackrel{(14) 3}{B}=\stackrel{(14) 3}{S}+\stackrel{(25) 3}{S}+\stackrel{(24) 4}{S}+\stackrel{(15) 4}{S}, \\
& \stackrel{(40) 4}{B}=\stackrel{(40) 4}{S}+\stackrel{(51) 4}{S}+\stackrel{(50) 5}{S}+\stackrel{(41) 5}{S}, \\
& \stackrel{(31) 4}{B}=\stackrel{(31) 4}{S}+\stackrel{(42) 4}{S}+\stackrel{(41) 5}{S}+\stackrel{(32) 5}{S}, \\
& \stackrel{(30) 1}{B}=\stackrel{(30) 1}{S}+\stackrel{(41) 1}{S}+\stackrel{(40) 2}{S}+\stackrel{(31) 2}{S}, \\
& \stackrel{(43) 3}{B}=\stackrel{(43) 3}{S}+\stackrel{(45) 3}{S}+\stackrel{444}{S}+\stackrel{(35) 4}{S}, \\
& \text { (24)4 (24) } 4 \quad 334 \quad \text { (23)5 } \\
& B=S+S+2 S \text {, } \\
& \begin{array}{lllll}
(32) 5 & \text { (32) } 5 & \text { (43)5 } & \text { (42)6 } & 336
\end{array} \\
& B=S+S+S+S \text {, } \\
& \stackrel{(21) 5}{B}=\stackrel{(21) 5}{S}+\stackrel{(32) 5}{S}+\stackrel{(31) 6}{S}+\stackrel{226}{S}, \\
& \stackrel{110}{B}=\stackrel{110}{S}+\stackrel{220}{S}+2 \stackrel{(12) 1}{S}, \\
& \stackrel{112}{B}=\stackrel{112}{S}+\stackrel{222}{S}+2 \stackrel{(12) 3}{S}, \\
& \stackrel{222}{B}=\stackrel{222}{S}+\stackrel{332}{S}+2 \stackrel{(32) 3}{S}, \\
& \stackrel{332}{B}=\stackrel{332}{S}+\stackrel{442}{S}+2 \stackrel{(43) 3}{S}, \\
& \stackrel{442}{B}=\stackrel{442}{S}+\stackrel{552}{S}+2 \stackrel{(54) 3}{S}, \\
& \stackrel{220}{B}=\stackrel{220}{S}+\stackrel{330}{S}+2 \stackrel{(32) 1}{S}, \\
& \stackrel{334}{B}=\stackrel{334}{S}+\stackrel{444}{S}+2 \stackrel{(43) 5}{S}, \\
& \stackrel{(14) 5}{B}=\stackrel{(14) 5}{S}+\stackrel{(25) 5}{S}+\stackrel{(24) 6}{S}+\stackrel{(15) 6}{S}, \\
& \stackrel{(43) 5}{B}=\stackrel{(43) 5}{S}+\stackrel{(54) 5}{S}+\stackrel{(53) 6}{S}+\stackrel{446}{S}, \\
& \stackrel{444}{B}=\stackrel{444}{S}+\stackrel{554}{S}+2 \stackrel{(54) 5}{S}, \\
& \stackrel{(14) 1}{B}=\stackrel{(14) 1}{S}+\stackrel{(25) 1}{S}+\stackrel{(24) 2}{S}+\stackrel{(15) 2}{S}, \\
& \stackrel{440}{B}=\stackrel{440}{S}+\stackrel{550}{S}+2 \stackrel{(54) 1}{S} \\
& \stackrel{(30) 5}{B}=\stackrel{(30) 5}{S}+\stackrel{(41) 5}{S}+\stackrel{(40) 6}{S}+\stackrel{(31) 6}{S},
\end{aligned}
$$




$$
\begin{aligned}
& \stackrel{(20) 4}{B}=\stackrel{(20) 4}{S}+\stackrel{(31) 4}{S}+\stackrel{(30) 5}{S}+\stackrel{(21) 5}{S} \text {, } \\
& \stackrel{(42) 0}{B}=\stackrel{(42) 0}{S}+\underset{S}{(53) 0} \quad \stackrel{(52) 1}{S}+\underset{S}{(43) 1} \\
& \begin{array}{lllll}
(40) 2 & (40) 2 & (51) 2 & (50) 3 \quad(41) 3
\end{array} \\
& B=S+S+S+S \text {, } \\
& \stackrel{(31) 0}{B}=\stackrel{(31) 0}{S}+\stackrel{(42) 0}{S}+\stackrel{(41) 1}{S}+\stackrel{(32) 1}{S} \text {, } \\
& \stackrel{(45) 1}{B}=\stackrel{(45) 1}{S}+\stackrel{552}{S}+\left(K_{4} \stackrel{(43) 1}{S}+K_{6} \stackrel{(41) 1}{S}+K_{6} \stackrel{(32) 1}{S}\right) \\
& +\frac{1}{2}\left(-\stackrel{552}{S}-K_{2} \stackrel{442}{S}+K_{4} \stackrel{332}{S}-2 K_{6} \stackrel{(31) 2}{S}+K_{6} \stackrel{222}{S}\right) \\
& \stackrel{(35) 2}{B}=\stackrel{(35) 2}{S}+\frac{1}{2}\left(-\stackrel{552}{S}-K_{2} \stackrel{442}{S}+K_{4} \stackrel{332}{S}-2 K_{6} \stackrel{(31) 2}{S}+K_{6} \stackrel{222}{S}\right)-K_{2} \stackrel{(43) 3}{S}+K_{6} \stackrel{(21) 3}{S} \text {, } \\
& \stackrel{(42) 6}{B}=\stackrel{(42) 6}{S}+\stackrel{(53) 6}{S}+\left(-K_{2} \stackrel{(52) 5}{S}-K_{4} \stackrel{(52) 3}{S}-K_{6} \stackrel{(52) 1}{S}\right)+\left(-K_{2} \stackrel{(43) 5}{S}-K_{4} \stackrel{(43) 3}{S}-K_{6} \stackrel{(43) 1}{S}\right) \text {, } \\
& \stackrel{336}{B}=\stackrel{336}{S}+\stackrel{446}{S}+2\left(-K_{2} \stackrel{(43) 5}{S}-K_{4} \stackrel{(43) 3}{S}-K_{6} \stackrel{(43) 1}{S}\right) \text {, } \\
& \stackrel{(25) 3}{B}=\stackrel{(25) 3}{S}-\stackrel{(54) 3}{S}-K_{2} \stackrel{(43) 3}{S}+K_{6} \stackrel{(21) 3}{S}+\stackrel{(53) 4}{S}+\frac{1}{2}\left(-\stackrel{444}{S}^{S}-2 K_{2} \stackrel{(42) 4}{S}-K_{4} \stackrel{224}{S}\right), \\
& \stackrel{446}{B}=\stackrel{446}{S}+\stackrel{556}{S}+2\left(-K_{2} \stackrel{(45) 5}{S}-K_{4} \stackrel{(45) 3}{S}-K_{6} \stackrel{(45) 1}{S}\right), \\
& \stackrel{(15) 4}{B}=\stackrel{(15) 4}{S}+\frac{1}{2}\left(-\stackrel{444}{S}-2 K_{2} \stackrel{(42) 4}{S}-K_{4} \stackrel{224}{S}\right)+\left(-\stackrel{(43) 5}{S}-K_{2} \stackrel{(41) 5}{S}-K_{2} \stackrel{(32) 5}{S}-K_{4} \stackrel{(21) 5}{S}\right) \text {, } \\
& \stackrel{(50) 5}{B}=\stackrel{(50) 5}{S}+\left(-\stackrel{(52) 5}{S}-\stackrel{(43) 5}{S}-K_{2} \stackrel{(41) 5}{S}-K_{2} \stackrel{(32) 5}{S}-K_{4} \stackrel{(21) 5}{S}\right)+\stackrel{(60) 6}{S}+\stackrel{(51) 6}{S} \\
& \stackrel{552}{B}=\stackrel{552}{S}+\left(K_{2} \stackrel{552}{S}+2 K_{4} \stackrel{(53) 2}{S}+2 K_{6} \stackrel{(51) 2}{S}+K_{4} \stackrel{442}{S}+2 K_{6} \stackrel{(42) 2}{S}+2 K_{4} \stackrel{332}{S}\right. \\
& \left.+K_{4} \stackrel{(43) 3}{S}+K_{6} \stackrel{(41) 3}{S}+K_{6} \stackrel{(32) 3}{S}\right) \\
& \stackrel{(54) 3}{B}=\stackrel{(54) 3}{S}+\left(K_{4} \stackrel{(43) 3}{S}+K_{6} \stackrel{(41) 3}{S}+K_{6} \stackrel{(32) 3}{S}\right)+\frac{1}{2}\left(\stackrel{554}{S}-K_{2} \stackrel{444}{S}+K_{4} \stackrel{334}{S}-2 K_{6} \stackrel{(31) 4}{S}+K_{6} \stackrel{224}{S}\right), \\
& \stackrel{(53) 4}{B}=\stackrel{(53) 4}{S}+\frac{1}{2}\left(-\stackrel{554}{S}-K_{2} \stackrel{444}{S}+K_{4} \stackrel{334}{S}-2 K_{6} \stackrel{(31) 4}{S}+K_{6} \stackrel{224}{S}\right)-K_{2} \stackrel{(43) 5}{S}+K_{6} \stackrel{(21) 5}{S}, \\
& \stackrel{(52) 5}{B}=\stackrel{(52) 5}{S}+\left(-\stackrel{(54) 5}{S}-K_{2} \stackrel{(43) 5}{S}+K_{6} \stackrel{(21) 5}{S}\right)+\stackrel{(53) 6}{S}+\frac{1}{2}\left(-\stackrel{446}{S}-2 K_{2} \stackrel{(42) 6}{S}-K_{4} \stackrel{226}{S}\right), \\
& \stackrel{(55) 4}{B}=\stackrel{(55) 4}{S}+\left(K_{2} \stackrel{554}{S}+2 K_{4} \stackrel{(53) 4}{S}+2 K_{6} \stackrel{(51) 4}{S}+K_{4} \stackrel{444}{S}+2 K_{6} \stackrel{(42) 4}{S}+K_{4} \stackrel{334}{S}\right) \\
& +2\left(K_{4} \stackrel{(43) 5}{S}+K_{6} \stackrel{(41) 5}{S}+K_{6} \stackrel{(32) 5}{S}\right)
\end{aligned}
$$




$$
\begin{aligned}
& \stackrel{(54) 5}{B}=\stackrel{(54) 5}{S}+\left(K_{4} \stackrel{(43) 5}{S}+K_{6} \stackrel{(41) 5}{S}+K_{6} \stackrel{(32) 5}{S}\right)+\stackrel{556}{S}+\frac{1}{2}\left(-\stackrel{554}{S}-K_{2} \stackrel{446}{S}+K_{4} \stackrel{336}{S}-2 K_{2} \stackrel{(31) 6}{S}+K_{6} \stackrel{226}{S}\right), \\
& \stackrel{330}{B}=\stackrel{330}{S}+\stackrel{440}{S}+2 \stackrel{(43) 1}{S}, \\
& \stackrel{(52) 1}{B}=\stackrel{(52) 1}{S}+\left(-\stackrel{(54) 1}{S}-K_{2} \stackrel{(43) 1}{S}+K_{6} \stackrel{(21) 1}{S}\right)+\stackrel{(53) 2}{S}+\frac{1}{2}\left(-\stackrel{442}{S}-2 K_{2} \stackrel{(42) 2}{S}-K_{4} \stackrel{222}{S}\right) \text {, } \\
& \stackrel{(15) 6}{B}=\stackrel{(15) 6}{S}+\frac{1}{2}\left(-\stackrel{446}{S}-2 K_{2} \stackrel{(42) 6}{S}-K_{4} \stackrel{226}{S}\right)+\left(-K_{4} \stackrel{(52) 3}{S}-K_{6} \stackrel{(52) 1}{S}\right) \\
& +\left(K_{2} \stackrel{(43) 5}{S}+K_{2} 2 \stackrel{(41) 5}{S}+K_{2} 2 \stackrel{(32) 5}{S}+K_{2} K_{4} \stackrel{(21) 5}{S}\right) \\
& +\left(K_{4} \stackrel{(52) 3}{S}+K_{4} \stackrel{(43) 3}{S}+K_{4} K_{2} \stackrel{(41) 3}{S}+K_{4} K_{2} \stackrel{(32) 3}{S}+K_{4} \stackrel{(21) 3}{S}^{2}\right) \\
& +\left(K_{6} \stackrel{(26) 1}{S}+K_{6} \stackrel{(43) 1}{S}+K_{6} K_{2} \stackrel{(41) 1}{S}+K_{6} K_{2} \stackrel{(32) 1}{S}+K_{6} K_{4} \stackrel{(21) 1}{S}\right), \\
& \stackrel{(51) 2}{B}=\stackrel{(51) 2}{S}+\frac{1}{2}\left(-\stackrel{(442)}{S}-2 K_{2} \stackrel{(42) 2}{S}-K_{4} \stackrel{222}{S}\right)+\left(-\stackrel{(43) 3}{S}-K_{2} \stackrel{(41) 3}{S}-K_{2} \stackrel{(32) 3}{S}-K_{4} \stackrel{(21) 3}{S}\right), \\
& \stackrel{(53) 6}{B}=\stackrel{(53) 6}{S}+\frac{1}{2}\left(-\stackrel{556}{S}-K_{2} \stackrel{446}{S}+K_{4} \stackrel{336}{S}-2 K_{6} \stackrel{(31) 6}{S}+K_{6} \stackrel{226}{S}\right) \\
& +K_{2}\left(K_{2} \stackrel{(43) 5}{S}+K_{4} \stackrel{(43) 3}{S}+K_{6} \stackrel{(43) 1}{S}\right)+K_{6}\left(-K_{2} \stackrel{(21) 5}{S}-K_{4} \stackrel{(21) 3}{S}-K_{6} \stackrel{(21) 1}{S}\right) \text {, } \\
& \stackrel{224}{B}=\stackrel{224}{S}+\stackrel{334}{S}+2 \stackrel{(23) 5}{S}, \\
& \stackrel{(55) 6}{B}=\stackrel{(55) 6}{S}+\left(K_{2} \stackrel{(556)}{S}+2 K_{4} \stackrel{(53) 6}{S}+2 K_{6} \stackrel{(51) 6}{S}+K_{4} \stackrel{446}{S}+2 K_{6} \stackrel{(42) 6}{S}+K_{4} \stackrel{336}{S}\right) \\
& +2 K_{4}\left[\left(-K_{2} \stackrel{(43) 5}{S}-K_{4} \stackrel{(43) 3}{S}-K_{6} \stackrel{(43) 1}{S}\right)+K_{6}\left(-K_{2} \stackrel{(41) 5}{S}-K_{4} \stackrel{(41) 3}{S}-K_{6} \stackrel{(41) 1}{S}\right)\right. \\
& \left.+K_{6}\left(-K_{2} \stackrel{(32) 5}{S}-K_{4} \stackrel{(32) 3}{S}-K_{6} \stackrel{(32) 1}{S}\right)\right], \\
& \stackrel{226}{B}=\stackrel{226}{S}+\stackrel{336}{S}+2\left(-K_{2} \stackrel{(32) 5}{S}-K_{4} \stackrel{(32) 3}{S}-K_{6} \stackrel{(32) 1}{S}\right) \text {, } \\
& \stackrel{(31) 6}{B}=\stackrel{(31) 6}{S}+\stackrel{(42) 6}{S}+\left(-K_{2} \stackrel{(41) 5}{S}-K_{4} \stackrel{(41) 3}{S}-K_{6} \stackrel{(41) 1}{S}\right)+\left(-K_{2} \stackrel{(32) 5}{S}-K_{4} \stackrel{(32) 3}{S}-K_{6} \stackrel{(32) 1}{S}\right) \text {, } \\
& \stackrel{(50) 3}{B}=\stackrel{(50) 3}{S}+\left(-\stackrel{(52) 3}{S}-\stackrel{(43) 3}{S}-K_{2} \stackrel{(41) 3}{S}-K_{2} \stackrel{(32) 3}{S}-K_{4} \stackrel{(21) 3}{S}\right) \\
& +\left(-K_{4} \stackrel{(20) 4}{S}-K_{2} \stackrel{(40) 4}{S}-2 \stackrel{(42) 4}{S}-K_{2} \stackrel{224}{S}\right)+\stackrel{(51) 4}{S}, \\
& \stackrel{(50) 1}{B}=\stackrel{(50) 1}{S}+\left(-\stackrel{(52) 1}{S}-\stackrel{(43) 1}{S}-K_{2} \stackrel{(41) 1}{S}-K_{4} \stackrel{(21) 1}{S}\right) \\
& +\left(-K_{4} \stackrel{(20) 2}{S}-K_{2} \stackrel{(40) 2}{S}-2 \stackrel{(42) 2}{S}-K_{2} \stackrel{222}{S}\right)+\stackrel{(51) 2}{S},
\end{aligned}
$$




$$
\begin{aligned}
& \stackrel{550}{B}=\stackrel{550}{S}+\left(K_{2} \stackrel{440}{S}+2 K_{4} \stackrel{(53) 0}{S}+2 K_{6} \stackrel{(51) 0}{S}+K_{4} \stackrel{440}{S}+2 K_{6} \stackrel{(42) 0}{S}+K_{4} \stackrel{330}{S}\right) \\
& +2\left(K_{4} \stackrel{(43) 1}{S}+K_{6} \stackrel{(41) 1}{S}+K_{6} \stackrel{(32) 1}{S}\right), \\
& \stackrel{(40) 6}{B}=\stackrel{(40) 6}{S}+\stackrel{(51) 6}{S}+\left(-K_{2} \stackrel{(50) 5}{S}-K_{4} \stackrel{(50) 3}{S}-K_{6} \stackrel{(50) 1}{S}\right)+\left(-K_{2} \stackrel{(41) 5}{S}-K_{4} \stackrel{(41) 3}{S}-K_{6} \stackrel{(41) 1}{S}\right) \text {, } \\
& \stackrel{(20) 6}{B}=\stackrel{(20) 6}{S}+\stackrel{(31) 6}{S}+\left(-K_{2} \stackrel{(30) 5}{S}-K_{4} \stackrel{(30) 3}{S}-K_{6} \stackrel{(30) 1}{S}\right)+\left(-K_{2} \stackrel{(21) 5}{S}-K_{4} \stackrel{(21) 3}{S}-K_{6} \stackrel{(21) 1}{S}\right) \text {, } \\
& \stackrel{(53) 0}{B}=\stackrel{(53) 0}{S}+\frac{1}{2}\left(-\stackrel{550}{S}-K_{2} \stackrel{440}{S}+K_{4} \stackrel{330}{S}-2 K_{6} \stackrel{(31) 0}{S}+K_{6}{ }^{220} S\right)+\left(-K_{2} \stackrel{(43) 1}{S}+K_{6} \stackrel{(21) 1}{S}\right) \text {, } \\
& \stackrel{(51) 0}{B}=\stackrel{(51) 0}{S}+\frac{1}{2}\left(-\stackrel{440}{S}-2 K_{2} \stackrel{(42) 0}{S}-K_{4} \stackrel{220}{S}\right)+\left(-\stackrel{(43) 1}{S}-K_{2} \stackrel{(41) 1}{S}-K_{2} \stackrel{(32) 1}{S}-K_{4} \stackrel{(21) 1}{S}\right) \text {. }
\end{aligned}
$$

Proof. Each equation of the system (6) may be obtained from [1, (3.14)], using [2, (25) and (2a)] in order. For example, the 55th equation of the system (6) follows as in the following way:

$$
\begin{aligned}
\stackrel{(53) 6}{B}= & \stackrel{(53) 6}{S}+\stackrel{(64) 6}{S}+\stackrel{(63) 7}{S}+\stackrel{(54) 7}{S} \\
= & \stackrel{(53) 6}{S}+\frac{1}{2}\left(-\stackrel{556}{S}-K_{2} \stackrel{446}{S}+K_{4} \stackrel{336}{S}+2 K_{6} \stackrel{(31) 6}{S}+K_{6} \stackrel{226}{S}\right) \\
& -\left(-K_{2} \stackrel{(54) 5}{S}-K_{4} \stackrel{(54) 3}{S}-K_{6} \stackrel{(54) 1}{S}\right)-K_{2}\left(-K_{2} \stackrel{(43) 5}{S}-K_{4} \stackrel{(43) 3}{S}-K_{6} \stackrel{(43) 1}{S}\right) \\
& +K_{6}\left(-K_{2} \stackrel{(21) 5}{S}-K_{4} \stackrel{(21) 3}{S}-K_{6} \stackrel{(21) 1}{S}\right)+\left(-K_{2} \stackrel{(54) 5}{S}-K_{4} \stackrel{(54) 3}{S}-K_{6} \stackrel{(54) 1}{S}^{(5)}\right) \\
= & \text { the right-hand side of the } 55 \text { th equation of the system (6). }
\end{aligned}
$$

THEOREM 3 (for the second class with the second category of 7-g-UFT). The system of equations $[1,(2.26)$ or (2.5)] is reduced to the following linear system of 25 equations:

$$
\begin{aligned}
& B=S+\stackrel{110}{S}+2 \stackrel{(10) 1}{S}, \\
& \stackrel{(10) 1}{B}=\stackrel{(10) 1}{S}+\stackrel{(21) 1}{S}+\stackrel{(20) 2}{S}+\stackrel{112}{S}, \\
& \stackrel{110}{B}=\stackrel{110}{S}+\stackrel{220}{S}+2 \stackrel{(12) 1}{S}, \\
& \stackrel{112}{B}=\stackrel{112}{S}+\stackrel{222}{S}+2 \stackrel{(21) 3}{S}, \\
& \stackrel{(21) 1}{B}=\stackrel{(21) 1}{S}+\stackrel{(32) 1}{S}+\stackrel{(31) 2}{S}+\stackrel{222}{S}, \\
& \stackrel{(20) 2}{B}=\stackrel{(20) 2}{S}+\stackrel{(31) 2}{S}+\stackrel{(30) 3}{S}+\stackrel{(21) 3}{S} \text {, }
\end{aligned}
$$




$$
\begin{aligned}
& \stackrel{222}{B}=\stackrel{222}{S}+\stackrel{332}{S}+2 \stackrel{(32) 3}{S}, \\
& \stackrel{220}{B}=\stackrel{220}{S}+\stackrel{330}{S}+2 \stackrel{(32) 1}{S}, \\
& \stackrel{(21) 3}{B}=\stackrel{(21) 3}{S}+\stackrel{(32) 3}{S}+\stackrel{(31) 4}{S}+\stackrel{224}{S}, \\
& \stackrel{(32) 1}{B}=\stackrel{(32) 1}{S}+K_{4} \stackrel{(21) 1}{S}+\frac{1}{2}\left(\stackrel{332}{S}-K_{2} \stackrel{222}{S}+K_{4} \stackrel{112}{S}\right), \\
& \stackrel{(31) 2}{B}=\stackrel{(31) 2}{S}-\frac{1}{2}\left(\stackrel{332}{S}+K_{2} \stackrel{222}{S}-K_{4} \stackrel{112}{S}\right)-K_{2} \stackrel{(21) 3}{S}, \\
& \stackrel{(30) 3}{B}=\stackrel{(30) 3}{S}-\stackrel{(32) 3}{S}-K_{2} \stackrel{(21) 3}{S}-K_{2} \stackrel{(20) 4}{S}-\frac{1}{2}\left(\stackrel{224}{S}+K_{4} \stackrel{004}{S}\right)+\stackrel{(31) 4}{S} \text {, } \\
& \stackrel{224}{B}=\stackrel{224}{S}+\stackrel{334}{S}-2 K_{2} \stackrel{(32) 3}{S}-2 K_{4} \stackrel{(32) 1}{S}, \\
& \stackrel{(32) 3}{B}=\stackrel{(32) 3}{S}+K_{4} \stackrel{(21) 3}{S}+\frac{1}{2}\left(\stackrel{334}{S}-K_{2} \stackrel{224}{S}+K_{4}{ }^{114} S\right), \\
& \stackrel{332}{B}=\left(1+K_{2}\right) \stackrel{332}{S}+K_{4}{ }^{222} S+2 K_{4} \stackrel{(31) 2}{S}+2 K_{4} \stackrel{(21) 3}{S}, \\
& \stackrel{330}{B}=\left(1+K_{2}\right) \stackrel{330}{S}+K_{4} \stackrel{220}{S}^{2}+2 K_{4} \stackrel{(31) 0}{S}+2 K_{4} \stackrel{(21) 1}{S}, \\
& \stackrel{(31) 4}{B}=\frac{1}{2}\left(-\stackrel{334}{S}-K_{2} \stackrel{224}{S}+K_{4} \stackrel{114}{S}\right)+\left(K_{2}\right) \stackrel{2}{2}{ }^{(21) 3}-K_{4} \stackrel{(21) 1}{S}, \\
& \stackrel{(20) 4}{B}=\stackrel{(20) 4}{S}+\stackrel{(31) 4}{S}-K_{2} \stackrel{(30) 3}{S}^{-K_{4}} \stackrel{(30) 1}{S}^{-K_{2}} \stackrel{(21) 3}{S}^{-K_{4}} \stackrel{(21) 1}{S} \text {, } \\
& \stackrel{334}{B}=\left(1+K_{2}\right) \stackrel{334}{S}+K_{4} \stackrel{224}{S}+2 K_{4} \stackrel{(31) 4}{S}-2 K_{4} K_{2} \stackrel{(21) 3}{S}-2\left(K_{4}\right) \stackrel{2(21) 1}{S}, \\
& \stackrel{114}{B}=\stackrel{114}{S}+\stackrel{224}{S}-2 K_{2} \stackrel{(21) 3}{S}-2 K_{4} \stackrel{(21) 1}{S}, \\
& \stackrel{004}{B}=\stackrel{004}{S}+\stackrel{114}{S}-2 K_{2} \stackrel{(10) 3}{S}-2 K_{4} \stackrel{(10) 1}{S}, \\
& \stackrel{(10) 3}{B}=\stackrel{(10) 3}{S}+\stackrel{(21) 3}{S}+\stackrel{(20) 4}{S}+\stackrel{114}{S}, \\
& \stackrel{002}{B}=\stackrel{002}{S}+\stackrel{112}{S}+2 \stackrel{(10) 3}{S}, \\
& \stackrel{(31) 0}{B}=\stackrel{(31) 0}{S}-\frac{1}{2}\left(\stackrel{330}{S}+K_{2} \stackrel{220}{S}-K_{4} \stackrel{110}{S}\right)-K_{2} \stackrel{(21) 1}{S} \\
& \stackrel{(30) 1}{B}=\stackrel{(30) 1}{S}-\stackrel{(32) 1}{S}-K_{2} \stackrel{(21) 1}{S}-K_{2} \stackrel{(20) 2}{S}-\frac{1}{2}\left(\stackrel{222}{S}+K_{4} \stackrel{002}{S}\right)+\stackrel{(31) 2}{S} \text {. }
\end{aligned}
$$

Proof. This assertion also follows from [1, (3.14)], using [2, (2b) and (26)]. 
THEOREM 4 (for the second class with the first category of 7-g-UFT). The system of $[1,(2.24)$ or (2.5)] is reduced to the following linear system of 5 equations:

$$
\begin{array}{rl}
B & =S+\stackrel{110}{S}+2 \stackrel{(10) 1}{S}, \\
\stackrel{110}{B} & =\left(1+K_{2}\right) \stackrel{110}{S}, \\
\stackrel{(10))}{B} & =\stackrel{(10) 1}{S}+\frac{1}{2}\left(\stackrel{112}{S}-K_{2} \stackrel{002}{S}\right), \\
{ }^{112} & =\left(1+K_{2}\right) \stackrel{112}{S}, \\
002 & 002+112 \\
B & S+2 K_{2} \stackrel{(10) 1}{S} .
\end{array}
$$

Proof. The system (9) may be obtained from [1, (3.14)], using [2, (2c) and (27)].

Each of the systems (6), (8), and (9) may be solved for $S=S_{\omega \lambda \mu}$ using the Gauss-Jordan elimination method. The systems (6) and (8), which contain many unknowns, may be solved by operating computer with the use of Mathematica version 4 . Since the solutions of (6) and (8) are too complicated, we just display the solution of the system (8) in the appendix and that of the system (9) in the following theorem.

THEOREM 5 (for the second class with the first category of 7-g-UFT). If condition (4) is satisfied, the unique solution of [1, (2.26)] may be given by

$$
\left[1-\left(K_{2}\right)^{2}\right](S-B)=1-\left(K_{2}\right)^{2}-\left(1-\left(K_{2}\right)^{2}\right) \stackrel{110}{B}-2 \stackrel{(10) 1}{B}+\stackrel{112}{B}-K_{2} \stackrel{002}{B} .
$$

Now that we have represented the tensor $S=S_{\omega \lambda \mu}$ in terms of $g_{\lambda \mu}$, it is possible for us to obtain the tensorial representation of $U_{\lambda \mu}^{v}$ and eventually the seven-dimensional Einstein's connection $\Gamma_{\lambda \mu}^{v}$ in terms of $g_{\lambda \mu}$ by simply substituting the respective representation of $S$ into [1, (2.23) and (2.22)].

\section{Appendix}

The solution of the system [1, (2.24) or (2.5)] for the case of the second class with the second category. After a long and tedious calculations by operating computer with the use of Mathematica version 4, we have the following theorem.

TheOREM A.1 (for the second class with the second category of 7-g-UFT). If condition (3) is satisfied, the unique solution of $[1,(2.24)$ or (2.5)] may be 
given by

$$
\begin{aligned}
\left(1+K_{2}+\right. & \left.K_{4}\right)^{2}\left(1-K_{2}+K_{4}\right)\left(1-K_{4}\right)\left\{\left(1-K_{2}+5 K_{4}\right)^{2}-4 K_{4}\left(K_{2}-2\right)^{2}\right\}(S-B) \\
= & -\left(1+K_{2}+K_{4}\right)\left\{\left(1-K_{2}+5 K_{4}\right)^{2}-4 K_{4}\left(K_{2}-2\right)^{2}\right\} * B+\phi_{1} \stackrel{(12) 1}{B} \\
& +\phi_{2} \stackrel{(23) 1}{B}+\phi_{3} \stackrel{(31) 2}{B}+\phi_{4} \stackrel{(12) 3}{B}+\phi_{5} \stackrel{(32) 2}{B}+\phi_{6} \stackrel{(31) 4}{B}+\phi_{7} \stackrel{112}{B}+\phi_{8} \stackrel{222}{B} \\
& +\phi_{9} \stackrel{332}{B}+\phi_{10} \stackrel{224}{B}+\phi_{11} \stackrel{114}{B}+\phi_{12} \stackrel{334}{B},
\end{aligned}
$$

where

$$
\begin{aligned}
& { }^{*} B=-1+K_{2}{ }^{2}-K_{4}-K_{2}{ }^{2} K_{4}+K_{4}{ }^{2}+K_{4}{ }^{3}+\left(K_{4}+K_{4}{ }^{2}\right) \stackrel{004}{B}+2 K_{2} K_{4} \stackrel{(10) 3}{B} \\
& +2 K_{2} K_{4} \stackrel{(30) 1}{B}+2 K_{2} \stackrel{(20) 4}{B}+K_{2} K_{4}{ }^{2} \stackrel{002}{B}+\left(1-K_{2}{ }^{2}+K_{4}+K_{2}{ }^{2} K_{4}-K_{2} K_{4}{ }^{2}\right) \stackrel{110}{B} \\
& +\left(2-2 K_{2}{ }^{2}+2 K_{4}+2 K_{2}{ }^{2} K_{4}\right) \stackrel{(10) 1}{B}+\left(2+2 K_{4}\right) \stackrel{(30) 3}{B}+\left(-1+K_{2}{ }^{2}-2 K_{4}-K_{4}{ }^{2}\right)^{220} \\
& +\left(-2+2 K_{2}{ }^{2}-2 K_{4}\right) \stackrel{(20) 2}{B}+\left(1-K_{2}+K_{4}\right) \stackrel{330}{B}+\left(-2 K_{4}+2 K_{2} K_{4}-2 K_{4}{ }^{2}\right) \stackrel{(31) 0}{B}, \\
& \phi_{1}=2\left(2-2 K_{2}-6 K_{2}{ }^{2}+3 K_{2}{ }^{3}+5 K_{2}{ }^{4}-K_{2}{ }^{5}-K_{2}{ }^{6}\right) \\
& +\left(-4-13 K_{2}+9 K_{2}{ }^{2}+13 K_{2}{ }^{3}+3 K_{2}{ }^{4}+4 K_{2}{ }^{5}\right) K_{4} \\
& +\left(-8-6 K_{2}-23 K_{2}{ }^{2}-46 K_{2}{ }^{3}-K_{2}{ }^{4}+4 K_{2}{ }^{5}\right) K_{4}{ }^{2} \\
& +\left(4+13 K_{2}-38 K_{2}{ }^{2}-20 K_{2}{ }^{3}-5 K_{2}{ }^{4}\right) K_{4}{ }^{3} \\
& +\left(6+18 K_{2}+16 K_{2}^{2}-2 K_{2}^{3}\right) K_{4}^{4}+10 K_{2} K_{4}{ }^{5}, \\
& \phi_{2}=2\left(-3+3 K_{2}+7 K_{2}{ }^{2}-5 K_{2}{ }^{3}-4 K_{2}{ }^{4}+2 K_{2}{ }^{5}\right) \\
& +\left(12+32 K_{2}-26 K_{2}{ }^{2}-42 K_{2}{ }^{3}+2 K_{2}{ }^{4}+2 K_{2}{ }^{5}\right) K_{4} \\
& +\left(38+37 K_{2}-41 K_{2}{ }^{2}-9 K_{2}{ }^{3}+K_{2}{ }^{4}\right) K_{4}{ }^{2} \\
& +\left(28+14 K_{2}+7 K_{2}{ }^{2}-2 K_{2}{ }^{3}\right) K_{4}{ }^{3}+\left(5+6 K_{2}-K_{2}{ }^{2}\right) K_{4}{ }^{4}, \\
& \phi_{3}=2\left(-3-K_{2}+6 K_{2}{ }^{2}+2 K_{2}{ }^{3}-3 K_{2}{ }^{4}-K_{2}{ }^{5}-8 K_{2}{ }^{6}\right)+\left(-8+7 K_{2}{ }^{2}+5 K_{2}{ }^{3}+4 K_{2}{ }^{4}\right) K_{4} \\
& +\left(-22-27 K_{2}-17 K_{2}{ }^{2}+7 K_{2}{ }^{3}+4 K_{2}{ }^{4}\right) K_{4}{ }^{2}+\left(-2+72 K_{2}+50 K_{2}{ }^{2}-8 K_{2}{ }^{3}\right) K_{4}{ }^{3} \\
& +\left(-5+29 K_{2}-4 K_{2}^{2}\right) K_{4}^{4}, \\
& \phi_{4}=2\left(-3-K_{2}+6 K_{2}{ }^{2}+2 K_{2}{ }^{3}-3 K_{2}{ }^{4}-K_{2}{ }^{5}\right)+\left(-8+7 K_{2}{ }^{2}+5 K_{2}{ }^{3}+4 K_{2}{ }^{4}\right) K_{4} \\
& +\left(-22-27 K_{2}-17 K_{2}{ }^{2}+7 K_{2}{ }^{3}+4 K_{2}{ }^{4}\right) K_{4}{ }^{2}+\left(-32-26 K_{2}-4 K_{2}{ }^{2}-2 K_{2}{ }^{3}\right) K_{4}{ }^{3} \\
& +\left(-15+2 K_{2}-2 K_{2}^{2}\right) K_{4}^{4} \text {, }
\end{aligned}
$$




$$
\begin{aligned}
& \phi_{5}=2\left(8+7 K_{2}-11 K_{2}{ }^{2}-11 K_{2}{ }^{3}+K_{2}{ }^{4}\right)+\left(8-15 K_{2}-9 K_{2}{ }^{2}+12 K_{2}{ }^{3}+2 K_{2}{ }^{4}\right) K_{4} \\
& +\left(-8-32 K_{2}+12 K_{2}{ }^{2}+9 K_{2}^{3}\right) K_{4}^{2}+\left(-8-15 K_{2}-6 K_{2}^{2}\right) K_{4}{ }^{3}-5 K_{2} K_{4}^{4}, \\
& \phi_{6}=2\left(4+K_{2}-7 K_{2}{ }^{2}-K_{2}{ }^{3}+3 K_{2}{ }^{4}\right)+\left(13+3 K_{2}-21 K_{2}{ }^{2}-7 K_{2}{ }^{3}\right) K_{4} \\
& +\left(71+59 K_{2}-2 K_{2}{ }^{2}-8 K_{2}^{3}\right) K_{4}^{2}+\left(87+57 K_{2}-4 K_{2}^{2}\right) K_{4}{ }^{3}+25 K_{4}^{4}, \\
& \phi_{7}=2-2 K_{2}-2 K_{2}{ }^{2}+4 K_{2}{ }^{3}-2 K_{2}{ }^{4}-2 K_{2}{ }^{5}+2 K_{2}{ }^{6} \\
& +\left(-2-14 K_{2}+16 K_{2}{ }^{2}+18 K_{2}{ }^{3}-12 K_{2}{ }^{4}-4 K_{2}{ }^{5}-2 K_{2}{ }^{6}\right) K_{4} \\
& +\left(14-11 K_{2}-K_{2}{ }^{2}+16 K_{2}^{3}+23 K_{2}{ }^{4}-5 K_{2}^{5}\right) K_{4}{ }^{2} \\
& +\left(42-34 K_{2}+19 K_{2}^{2}+71 K_{2}^{3}-6 K_{2}^{4}\right) K_{4}^{3} \\
& +\left(24-77 K_{2}+18 K_{2}{ }^{2}-3 K_{2}^{3}\right) K_{4}{ }^{4}-10 K_{2} K_{4}{ }^{5}, \\
& \phi_{8}=-6+20 K_{2}{ }^{2}+4 K_{2}{ }^{3}-18 K_{2}{ }^{4}-4 K_{2}{ }^{5}+4 K_{2}{ }^{6} \\
& +\left(4+59 K_{2}+37 K_{2}{ }^{2}-90 K_{2}{ }^{3}-73 K_{2}{ }^{4}+3 K_{2}{ }^{5}+4 K_{2}{ }^{6}\right) K_{4} \\
& +\left(16+127 K_{2}+87 K_{2}{ }^{2}-45 K_{2}{ }^{3}-23 K_{2}{ }^{4}-2 K_{2}{ }^{5}\right) K_{4}{ }^{2} \\
& +\left(-4+101 K_{2}+77 K_{2}{ }^{2}+9 K_{2}{ }^{3}-2 K_{2}{ }^{4}\right) K_{4}{ }^{3}+\left(-10+33 K_{2}+7 K_{2}{ }^{2}\right) K_{4}{ }^{4}, \\
& \phi_{9}=6-6 K_{2}-14 K_{2}{ }^{2}+10 K_{2}{ }^{3}+8 K_{2}{ }^{4}-4 K_{2}{ }^{5} \\
& +\left(-14-53 K_{2}+32 K_{2}{ }^{2}+72 K_{2}{ }^{3}-K_{2}{ }^{4}-4 K_{2}{ }^{5}\right) K_{4} \\
& +\left(-46-86 K_{2}+41 K_{2}{ }^{2}+20 K_{2}{ }^{3}+2 K_{2}{ }^{4}\right) K_{4}{ }^{2} \\
& +\left(-26-17 K_{2}-17 K_{2}{ }^{2}+2 K_{2}{ }^{3}\right) K_{4}{ }^{3}-10 K_{2} K_{4}{ }^{4}, \\
& \phi_{10}=5+15 K_{2}-4 K_{2}{ }^{2}-23 K_{2}{ }^{3}-5 K_{2}{ }^{4}+4 K_{2}{ }^{5} \\
& +\left(40+44 K_{2}-71 K_{2}{ }^{2}-68 K_{2}{ }^{3}+3 K_{2}{ }^{4}+4 K_{2}{ }^{5}\right) K_{4} \\
& +\left(90+88 K_{2}-28 K_{2}{ }^{2}-19 K_{2}{ }^{3}-2 K_{2}{ }^{4}\right) K_{4}{ }^{2} \\
& +\left(80+64 K_{2}+7 K_{2}{ }^{2}-2 K_{2}{ }^{3}\right) K_{4}{ }^{3}+\left(25+5 K_{2}\right) K_{4}^{4}, \\
& \phi_{11}=K_{4}\left(-11-7 K_{2}+16 K_{2}{ }^{2}+13 K_{2}{ }^{3}-K_{2}{ }^{4}-2 K_{2}{ }^{5}\right) \\
& +\left(-25-2 K_{2}+33 K_{2}{ }^{2}+15 K_{2}{ }^{3}-K_{2}{ }^{4}\right) K_{4} \\
& +\left(-49+33 K_{2}+46 K_{2}{ }^{2}-6 K_{2}{ }^{3}\right) K_{4}{ }^{2}+\left(-35+28 K_{2}-3 K_{2}{ }^{2}\right) K_{4}{ }^{3} \text {, } \\
& \phi_{12}=-9-12 K_{2}+20 K_{2}{ }^{2}+13 K_{2}{ }^{3}-4 K_{2}{ }^{4}+\left(-35+9 K_{2}+50 K_{2}{ }^{2}-K_{2}{ }^{3}-4 K_{2}{ }^{4}\right) K_{4} \\
& +\left(-11+14 K_{2}+2 K_{2}{ }^{2}\right) K_{4}{ }^{2}+\left(15-7 K_{2}+2 K_{2}{ }^{2}\right) K_{4}{ }^{3} .
\end{aligned}
$$


ACKNOWLEDGMENT. This work was partially supported by the Basic Science Research Institute Program, Ministry of Education, Korea, 1997, BSRI-971442.

\section{REFERENCES}

[1] M. A. Kim, K. S. So, C. H. Cho, and K. T. Chung, Seven-dimensional considerations of Einstein's connection. II. The recurrence relations of the second and third kind in 7-g-UFT, Int. J. Math. Math. Sci. 2003 (2003), no. 14, 895-902.

[2] - Seven-dimensional considerations of Einstein's connection. I. The recurrence relations of the first kind in $n-g-U F T$, Int. J. Math. Math. Sci. 2003 (2003), no. $12,777-787$.

Mi Ae Kim: Department of Mathematics, Yonsei University, Seoul 120-749, Korea

Keum Sook So: Department of Mathematics, Hallym University, Chunchon 200-702, Korea

E-mail address: ksso@sun.ha11ym.ac.kr

Chung Hyun Cho: Department of Mathematics, Inha University, Inchon 402-751, Korea

E-mail address: chcho@math.inha.ac.kr

Kyung Tae Chung: Department of Mathematics, Yonsei University, Seoul 120-749, Korea

E-mail address: ktchung@yonsei . ac.kr 


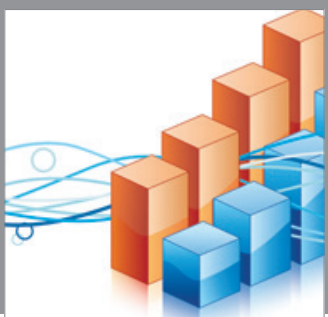

Advances in

Operations Research

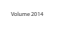

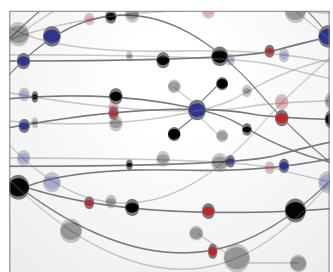

\section{The Scientific} World Journal
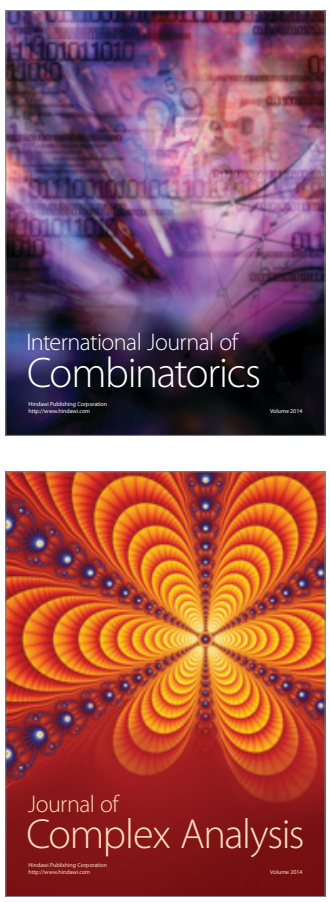

International Journal of

Mathematics and

Mathematical

Sciences
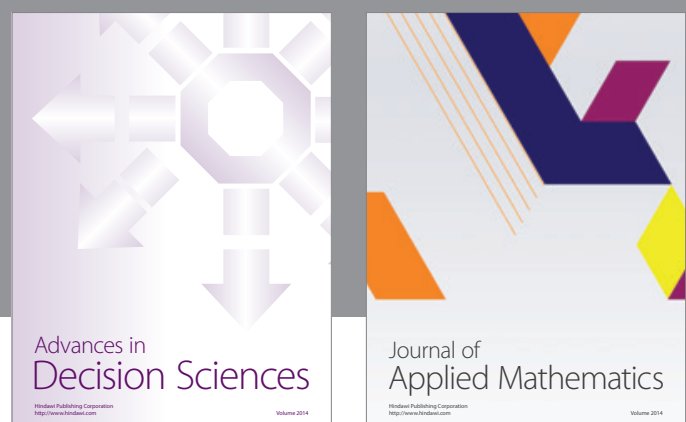

Journal of

Applied Mathematics
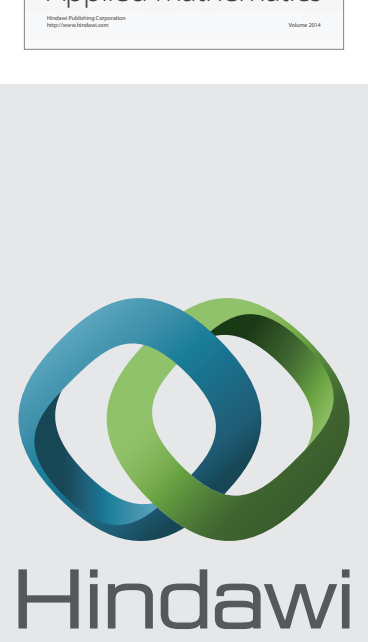

Submit your manuscripts at http://www.hindawi.com
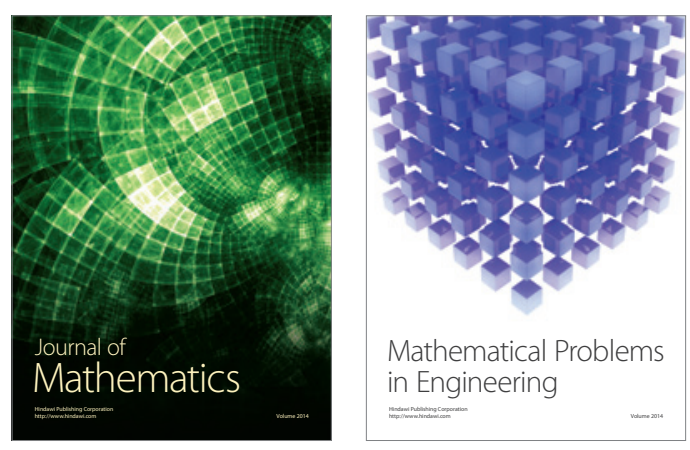

Mathematical Problems in Engineering
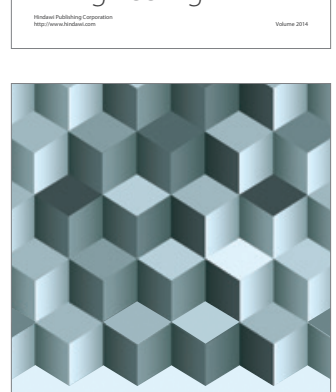

Journal of

Function Spaces
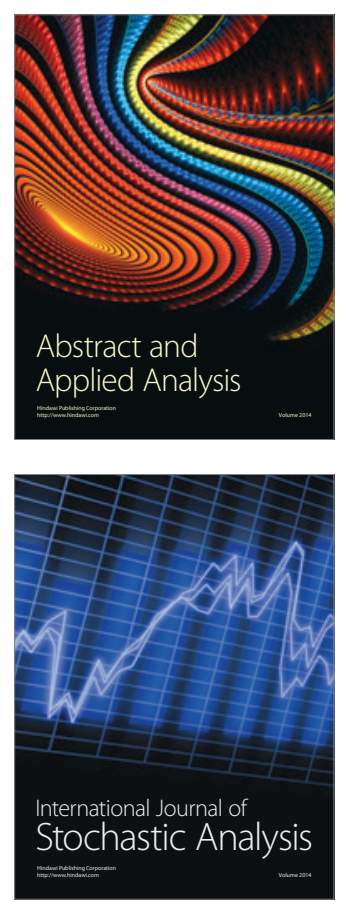

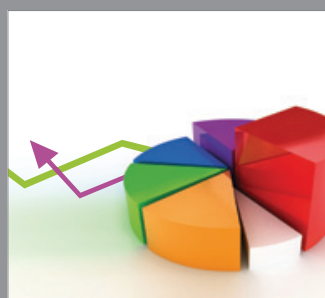

ournal of

Probability and Statistics

Promensencen
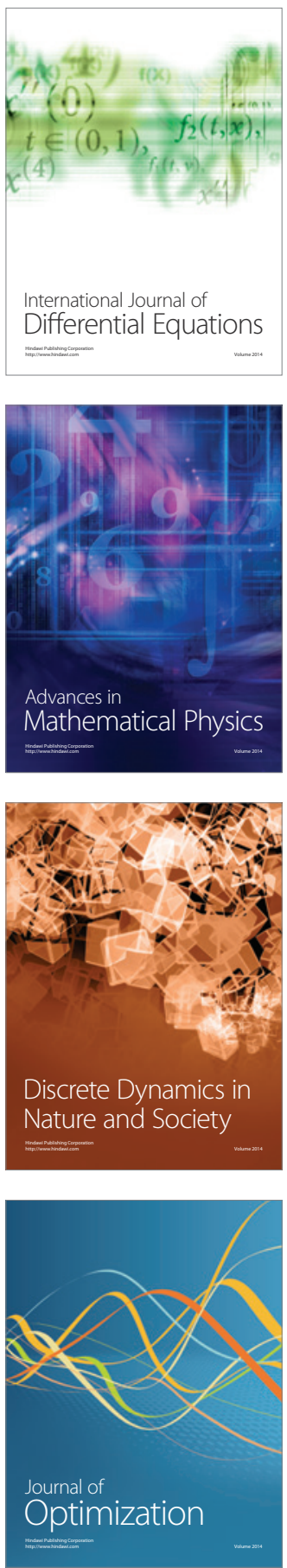